\title{
An Improving Densification in Spark Plasma Sintering Ultrafine-grained Y203 Transparent Ceramic by Particle Fracture and Rearrangement
}

\section{Zhangyi Huang}

Chengdu University

\section{Yang Shi}

Chengdu University

\section{Yutong Zhang}

Sichuan University

Mao Deng

Chengdu University

Haomin Wang

Chengdu University

Yi Guo

Chengdu University

Qingquan Kong

Chengdu University

Jianqi Qi

Sichuan University

\section{Benyuan Ma}

Huizhou University

Qingyuan Wang ( $\square$ wangqy@scu.edu.cn)

Chengdu University

\section{Research Article}

Keywords: Transparent ceramic, Nanorods, Fracture and rearrangement, Spark plasma sintering, Y203

Posted Date: October 5th, 2021

DOl: https://doi.org/10.21203/rs.3.rs-940172/v1

License: (c) (i) This work is licensed under a Creative Commons Attribution 4.0 International License.

Read Full License 


\section{Abstract}

Herein, we report a new strategy on an improving densification in spark plasma sintering (SPS) ultrafinegrained $\mathrm{Y}_{2} \mathrm{O}_{3}$ transparent ceramic using anisotropic-shaped nanorod powders. At the low temperature stage $\left(<600^{\circ} \mathrm{C}\right)$, fracture and rearrangement of nanorod powders will appear which can further improve the initial density of particle compact and reduce the initial particle size. When the temperature reaches beyond $600^{\circ} \mathrm{C}$, the sintering efficiency of nanorod powder compact will exceed that of the green body packed by spherical powders. The ceramic sample sintered at $1300^{\circ} \mathrm{C}$ from nanorods is transparent with ultra-fine grain and shows good optical and mechanical properties, while the corresponding ceramic from near-spherical nanocrystalline powders is not dense enough and opaque.

\section{Introduction}

Transparent ceramics can find a wide range of applications, such as transparent armors, infrared (IR) domes/windows, host material for fluorescence application, and laser hosts, on account of their processing flexibility in fabricating large-sized or complex-shaped products [1-6]. In most cases, the preparation process of any ceramics is sintering the consolidated/packed powders at high temperatures [7]. Therefore, the properties of the powder significantly influence the microstructures and performances of transparent ceramics.

As highly transparent ceramics require full density, well-reputed wisdom believes that ceramic powders with high sintering efficiency for obtaining highly transparent ceramics have some important characteristics including small crystallite size, narrow size distribution, spherical/near-spherical shape, and low-degree agglomeration [7]. It is well known that the surface energy of starting powders is serving as the driving force for densification during the sintering process $[8,9]$. Thus, the smaller the crystallite/particle size of the initial powders, the higher the densification rate [7]. Besides, spherical nanoparticles with narrow size distribution guarantee homogeneous packing and low pore-to-particle-size ratio in the ceramic green body [10]. Mono-dispersed powders can avoid large pores between agglomerates and forming crack-like voids in the final ceramics $[11,12]$. Fallaciously, achieving these aforementioned desirable characteriastics for powders faces a sea of troubles. For example, addressing agglomeration of the powders, especially for nanocrystalline powders, is a remaining challenge as nanoparticles tend to agglomerate to reduce surface energy due to the minimum energy principle [13, 14]. Agglomeration will directly result in heterogeneity of the packed green bodies, which will cause a phenomenon referred to as differential sintering [15]. It is inspiring that anisotropic-shaped powders, rather than spherical/near-spherical powders, can improve the densification process and provide some new densifying routes[16, 17]. For example, with the aid of high pressure (5 GPa), $\mathrm{Y}_{2} \mathrm{O}_{3}$ nanorod powder compact can achieve near full density through particle fracture, rearrangement, deformation, and interface sliding at only $500^{\circ} \mathrm{C}$ without atomic diffusion [16]. However, most sintering methods, such as spark plasma sintering (SPS), vacuum sintering, and hot isostatic pressure (HIP) sintering cannot provide such a high pressure[1, 7]. In addition, it is still an unrevealed controversy that whether anisotropicshaped nanorod powders are better than spherical/near-spherical powders supported by conventional 
wisdom in terms of sintering efficiency in these conventional sintering methods with dominated densification mechanisms of atomic diffusion. If they are suitable for preparing transparent ceramics, is there a sintering mechanism different from that of spherical/near spherical powders?

To address these questions, we have tried to use a solvothermal method to synthesize $\mathrm{Y}_{2} \mathrm{O}_{3}$ nanocrystalline powders with singularly shaped nanoparticles. As a comparison for checking sintering efficiency, near-spherical $\mathrm{Y}_{2} \mathrm{O}_{3}$ nanocrystalline powders were also synthesized by a co-precipitation method. Then, SPS was employed to sinter ceramics at various temperatures from 25 to $1400^{\circ} \mathrm{C}$. Specific surface areas and microstructures of these powders, as well as the relative densities, microstructures, and grain sizes of the sintered ceramics, were analyzed. The sintering efficiency and densification mechanism of the nanorods powders were investigated. Furthermore, the optical and mechanical properties of the $\mathrm{Y}_{2} \mathrm{O}_{3}$ transparent ceramic obtained at the optimized sintering temperature were analyzed.

\section{Material And Methods}

Herein, the $\mathrm{Y}_{2} \mathrm{O}_{3}$ nanorod powders were synthesized by a solvothermal method. $\mathrm{Y}\left(\mathrm{NO}_{3}\right)_{3} \cdot 6 \mathrm{H}_{2} \mathrm{O}(>99.99 \%$, Yutai Qixin Chemical Co. Ltd, China) was dissolved in deionized water to a concentration of $0.1 \mathrm{~mol} / \mathrm{L}$. Then, the solution was added drop by drop into the diluted ammonia solution $(0.5 \mathrm{~mol} / \mathrm{L})$. The mixed solutions were stirred for $6 \mathrm{~h}$ and aged for $20 \mathrm{~h}$ to get gel-like precipitate. After that, the precipitate was washed by deionized water and ethanol in turn several times using a centrifugal machine. The precipitate after drying and calcination can obtain nanocrystalline powders composed of spherical particles. To prepare singularly-shaped nanocrystalline powders, the washed precipitate was subsequently diluted by ethanol and placed into a Teflon reactor to react at $200^{\circ} \mathrm{C}$ for $24 \mathrm{~h}$. The reactants were washed centrifugally with ethanol and then dried at $60^{\circ} \mathrm{C}$. Finally, the as-obtained precursor powders were sieved using a 200-mesh screen and calcined at $800^{\circ} \mathrm{C}$ to remove the adsorbed water and bound water. Nearspherical powders were fabricated by directly dring the washed precipitate, sieving, and calcinating the precursor powders at $800^{\circ} \mathrm{C}$.

The as-prepared powders were placed into a graphite die with an inner diameter of $10 \mathrm{~mm}$. The densification processes of the samples were carried out in an SPS system (LABOX-325, Sinter Land, Japan) with an ambient pressure of $6 \mathrm{~Pa}$. A uniaxial pressure of $50 \mathrm{MPa}$ was applied on the $\mathrm{Y}_{2} \mathrm{O}_{3}$ samples during the sintering process. The samples are sintered at the temperature range of $25-1400^{\circ} \mathrm{C}$ with a ramping and cooling rate of $20^{\circ} \mathrm{C} / \mathrm{min}$, and hold for $5 \mathrm{~min}$ at the desired temperatures. Postannealing of the SPSed samples was conducted at $1000^{\circ} \mathrm{C}$ for $8 \mathrm{~h}$ in air using a muffle furnace. The asobtained samples were mirror polished for both two sides for in-line transmittance and Vickers hardness tests.

The crystal structure of the as-synthesized powders was investigated by X-ray diffraction using an X-ray diffractometer (Bruker D8 Advance, Bruker Co., German). The XRD data was recorded over the $2 \theta$ range of $10^{\circ}-70^{\circ}$ with a step size of $0.02^{\circ}$. High-magnified microstructural images of the powders and ceramics were checked by transmission electron microscopy (TEM, F200, JOEL, Tokyo, Japan). The fracture 
surfaces of the ceramics sintered at different temperatures were investigated by scanning electron microscope (SEM, JIB-4700F, JOEL, Tokyo, Japan). Specific surface areas of nanocrystalline powders were measured by a multipoint Brunauer Emmett Teller (BET) method (Tristar 3000, Micromeritics, Atlanta) using $\mathrm{N}_{2}$ as the adsorbate gas. The in-line transmittance test was performed on a UV-VIS-NIR spectrophotometer (Lamda 750, Perkin Elmer, USA). Vickers hardness tests were operated at an applied load of $0.5 \mathrm{~N}$ for $15 \mathrm{~s}$ (DUH-211s, Shimadzu, Japan). At least 10 measurements were carried out on each sample.

\section{Results And Discussion}

Fig. $1 \mathrm{~A}$ shows the XRD pattern of the synthesized $\mathrm{Y}_{2} \mathrm{O}_{3}$ powders. The pattern is well-indexed to the $\mathrm{Y}_{2} \mathrm{O}_{3}$ phase (PDF\#43-1036). Broad diffraction peaks indicate that the crystallite size of the powders may be in nanoscale. After the whole pattern fitting on the XRD data using the JADE software (Fitting error $<10 \%$ ), the average crystallite size of the calcined powder is calculated from peak broadening of XRD pattern using the Scherrer's equation [18], $D=0.89 \lambda / \beta \cos \theta$, where $D$ is the crystallite size, $\lambda$ value is $1.5406 \AA, \theta$ is the angle of the peak maximum (in $2 \theta$ ) and $\beta$ is the full width at half maximum (in $2 \theta$ ). From the calculation, the average crystallite size is $19.8 \pm 0.6 \mathrm{~nm}$. Fig. 1B displays the microstructure of the asprepared powders after calcination under $800^{\circ} \mathrm{C}$. The $\mathrm{Y}_{2} \mathrm{O}_{3}$ powders after solvothermal reaction and calcination present a rod-like morphology with low agglomeration and well-dispersed characteristics (Fig. 1B). The length of the nanorods is about $500 \mathrm{~nm}$. Previous studies have shown that well-dispersed nanocrystalline powders can supply abundant surface energy to promote ceramic densification [19-21]. Thus, the as-obtained nanorod powders may have good sinterability. The formation mechanism of anisotropically shaped nanorods rather than spherical nanoparticles may be induced by the competition between crystallographic anisotropy and crystallite growth kinetics in the solvothermal reaction process [22]. Fig. $1 \mathrm{C}$ shows the microstructure for a monodispersed nanorod. The diameter of the nanorod is about $20 \mathrm{~nm}$, which is consistent with the average crystallite size calculated from XRD data. Fig.1D-E present the HRTEM images taken from different areas in Fig. 1C. We can observe that a nanorod is composed of randomly oriented polycrystallines.

Fig. $2 \mathrm{~A}$ and Fig. $3 \mathrm{~A}$ show that the green body from nanorod powders compacted at $50 \mathrm{MPa}$ possessing a relative density of $36.7 \%$ is composed of randomly and loosely distributed nanorods. Fig. 2B-I reveal the microstructural evolutions of sintered ceramics from nanorod powders during sintering. As the sintering temperature reaches $900^{\circ} \mathrm{C}$, the nanorods are completely disappeared and near-spherical grains were observed. When the sintering temperature is in the range of $900-1200{ }^{\circ} \mathrm{C}$, residual pore significantly decreases and bulk density gradually increases with the elevation of the sintering temperature (Fig. 2B-E and Fig. 3). When the temperature $\geq 1250^{\circ} \mathrm{C}$, pores almost disappeared and ceramics with relative density higher than $98.8 \%$ exhibit a dense structure (Fig. 2F-I and Fig. 3). It is worth noting that when the sintering temperature is higher than $1300^{\circ} \mathrm{C}$, the density begins to decrease slightly. This phenomenon that the density decreases with the grain growth is common in the pressure-assisted sintering process $[23,24]$. The process of grain growth easily induces pore growth and non-uniform grains. Few fusion- 
grown pores lead to a decrease in density [25]. In addition, we can see from Fig. 2 that the grain size of ceramic increases with the increase of sintering temperature. We use Nanomeasure software to statistically count the average grain size of ceramics from Fig. 2. Grains with obvious and distinguishable boundaries for selected statistics. As the grains of ceramics sintered at relatively low temperatures (200$1000^{\circ} \mathrm{C}$ ) are difficult to distinguish, XRD patterns of these ceramics with no polishing were recorded. Whole patter fitting on XRD patterns with fitting errors $<7 \%$ were conducted for calculating the average grain size. The broaden peaks (Fig. 3B) indicate that the grain sizes of these ceramics are in nanoscale. FWHMs of diffraction peaks of (222), (400), and (440) decrease with the increase of sintering temperature (Fig. $3 \mathrm{C}$ ), indicating that grain starts to grow slowly after $>400{ }^{\circ} \mathrm{C}$. Image-based statistical and XRD calculated results show that the average grain size is less than $100 \mathrm{~nm}$ when the sintering temperature is $<1200^{\circ} \mathrm{C}$, and is at the sub-micron scale when the sintering temperature is $\leq 1300^{\circ} \mathrm{C}$. As the temperature rises to $1350^{\circ} \mathrm{C}$, the average grain size will increase to $1.9 \mu \mathrm{m}$ (Fig. 3D). $1350{ }^{\circ} \mathrm{C}$ is the $T_{g}$ (the critical temperature to trigger significant grain growth) of the $\mathrm{Y}_{2} \mathrm{O}_{3}$ specimen in this work as the average grain size increases from 1.9 to $38 \mu \mathrm{m}$ when the sintering temperature is elevated from 1350 to $1400{ }^{\circ} \mathrm{C}$. It can be concluded that $1300^{\circ} \mathrm{C}$ is the optimized sintering temperature for $\mathrm{Y}_{2} \mathrm{O}_{3}$ nanorod powders as the sintered ceramic reaches near-full density relative density with the average grain size is only $0.7 \mu \mathrm{m}$. Besides, the corresponding ceramic after polishing is highly transparent. Its optical property is discussed in the next part.

To investigate the powder effect on the sintering, near-spherical nanoparticles with an average crystallite size of about $21 \mathrm{~nm}$ (see Fig. S1 in the supplementary file) were also densified at various temperatures. It can be seen from Figure $3 \mathrm{~b}$ that when the temperature is $\leq 400^{\circ} \mathrm{C}$, the density of the ceramic from the near-spherical powders is higher than that of the ceramic from nanorod powders. However, as the temperature reached $600^{\circ} \mathrm{C}$, the density of the ceramic prepared by the nanorod powders increased significantly, which is higher than the density of the ceramic from the near-spherical powders. In the optimal sintering condition $\left(1300^{\circ} \mathrm{C}\right)$ for nanorod powders, the ceramic from near-spherical powders is completely opaque with many submicron-sized pores dwelled between grains (see Fig. S2 in the supplementary file). To elucidate the superiority of the nanorod powders over the near-spherical nanocrystalline powder in terms of sinterability, specific surface areas of these two kinds of powders were measured. The results show that the BET surface area of the nanorod powders is $67 \mathrm{~m}^{2} / \mathrm{g}$, higher than that of the spherical nanoparticles $\left(43 \mathrm{~m}^{2} / \mathrm{g}\right)$. This increase in BET surface area is mainly due to that ethanol can effectively break up the structures of the hydrated hydroxide, effectively eliminating agglomerations caused by hydrogen bond [21]. Thus, the near-full densification of nanorod powder achieved at only $1300^{\circ} \mathrm{C}$ is promoted by the high specific surface area based on the well-reputed wisdom that surface energy provides a driving force for the densification of ceramic.

Furthermore, we have noticed that $600{ }^{\circ} \mathrm{C}$ is a breakthrough point for the increase in the density of nanorod powders. There may be new mechanisms in the sintering process. In the process of preparing transparent ceramics by high-pressure sintering, the nano-particles will yield under high temperature and high-pressure conditions, which will cause the microscopic residual stress and strain of the bulk piece to 
decrease $[26,27]$. The experimental results showed that the yttrium oxide nanorods were fractured and rearranged under the conditions of $5 \mathrm{GPa} / 500^{\circ} \mathrm{C}$ [16]. It can be observed in Figure $2 \mathrm{~B}$ that the nanorods have completely disappeared at $900^{\circ} \mathrm{C}$, and the ceramic grains have not grown significantly (remaining at the nanometer level). Therefore, the nanorods are likely fractured under the pressure of 50MPa and high temperature. We consider that the fracture of nanorods will also cause the microscopic residual stress from the uncoordinated deformation of particles during consolidation. As microscopic deviatoric strain will lead to the X-ray line broadening in addition to reduced grain size $[26,28]$, we calculate the residual strain through the XRD data after the whole spectrum fitting. According to the well-reputed WilliamsonHall model $[28,29]$ and its corresponding variations, the residual micro-strain $(\varepsilon)$ of the specimen can be defined as:

$$
\Delta d_{o b s}^{2}=\Delta d_{i n s}^{2}+\Delta d_{s i z e}^{2}+\varepsilon^{2} d^{2}(P, T)
$$

where $\Delta \mathrm{d}_{\text {obs }}$ is the FWHM, $\Delta \mathrm{d}_{\text {ins }}$ the peak width at a stress-free state, $\Delta \mathrm{d}_{\text {size }}$ the peak width of grain size and $\mathrm{d}(\mathrm{P}, \mathrm{T})$ the $d$ spacing of a given lattice plane which is incorporated in the Scherrer formula. During the calculations, we subtract the instrument resolution and don't disjoin the changes in the peak width of the various contributions. Therefore, the micro-strain can be denoted as follows:

$$
\varepsilon=\sqrt{\Delta d_{o b s}^{2}-\Delta d_{\text {ins }}^{2}} / d(P, T)
$$

The calculated results show that the residual micro-strain increases with the increase of temperature and reaches the maximum value at $400^{\circ} \mathrm{C}$. As the temperature continues to increase, the residual strain decreases rapidly and tends to a stable value after $800^{\circ} \mathrm{C}$ (Fig. 4). To elucidate the mechanism of microstrain variations, TEM images of ceramic samples sintered at $200-900{ }^{\circ} \mathrm{C}$ were recorded. The results show that when the sintering temperature is $\leq 400^{\circ} \mathrm{C}$, the sample is mainly composed of nanorod particles. As the temperature reaches $600^{\circ} \mathrm{C}$, most nanorods disappear, and the length of remaining nanorods is greatly reduced. Near-spherical grains with a size of about $20 \mathrm{~nm}$ can be observed. Therefore, we can conclude that with increasing temperature, the fracture strength decreases and the nanorod particles fracture. When the temperature reaches $800^{\circ} \mathrm{C}$, the nanorods completely disappear, and the average grain size has grown to about $30 \mathrm{~nm}$. As the temperature continues to increase, the grains grow further. Thus, the SPS process of the nanorod powders can be divided into three stages with the sintering temperature increases, as shown in the schematic in Fig.4.. The first stage is the deviatoric-stressaccumulation stage. Then, nanorods begin to fracture and rearrange in the second stage. The stress begins to release and the strain decreases significantly. After that, grains growth is triggered. This stage is equivalent to the intermediate sintering stage in most ceramic sintering methods as the relative density of the corresponding ceramic sintered at $800^{\circ} \mathrm{C}$ is about 77.2\% [7]. Based on the aforementioned results, we consider that the fracture and rearrangement of nanorods under modest temperature and pressure also contributes to the high sintering efficiency of nanorods powders as it will further increase the surface area of the powders and consolidated density of ceramics. 
$\mathrm{Y}_{2} \mathrm{O}_{3}$ is an attractive optical material using as infrared dome and laser host [30]. As $\mathrm{Y}_{2} \mathrm{O}_{3}$ transparent ceramic was successfully obtained from nanorods powders, the in-line transmittance spectra and the optical image of the ceramic sintered at $1300{ }^{\circ} \mathrm{C}$ is presented in Fig. 5 . It can be seen from the optical image that the sample sintered under the optimized condition is highly transparent with maximum transmittances of $65.9 \%$ and $80.8 \%$ in the visible light and infrared wavelength, respectively. The relatively lower transparency in the visible light band than the infrared band indicates the light scattering. Based on the well-established light-scattering model [31, 32], the main source for light scattering may be assigned to a similar size between the average grain and the visible light wavelength. Generally, transparent ceramics prepared by SPS tend to appear yellow or brown colors, which is mainly caused by the absorption of visible light by dislocations or oxygen vacancies formed during the sintering process [33-35]. The as-obtained $\mathrm{Y}_{2} \mathrm{O}_{3}$ transparent ceramic is colorless, indicating near no dislocations or oxygen vacancies inside the transparent ceramic sample after annealing. Table 1 representatively lists the maximum transmittances of $\mathrm{Y}_{2} \mathrm{O}_{3}$ transparent ceramics prepared by SPS in the visible light and infrared band. It can be seen from Table 1 that the transmittances of $\mathrm{Y}_{2} \mathrm{O}_{3}$ transparent ceramics via SPS method in the visible light band are difficult to exceed $80 \%$, which are generally lower than the transmittances in the infrared range. The sintering temperatures to prepare $\mathrm{Y}_{2} \mathrm{O}_{3}$ transparent ceramics with good optical properties in most cases need to exceed $1400{ }^{\circ} \mathrm{C}$ [36-39]. However, when the nanorod powders were used in the present work, transparent ceramic with a transmittance of more than $80 \%$ in the infrared band was obtained at only $1300{ }^{\circ} \mathrm{C}$. Moreover, the transmittance of $\mathrm{Y}_{2} \mathrm{O}_{3}$ transparent ceramic obtained at $1300{ }^{\circ} \mathrm{C}$ as reported in other work is only $62.1 \%$ in the infrared band [39]. Although a higher thickness of the sample will reduce the transparency, it has no transmittance in the visible light band. To sum up, the assynthesized nanorod powders exhibit desirable sinterability and facilitate obtaining $\mathrm{Y}_{2} \mathrm{O}_{3}$ transparent ceramic with good optical properties at a relatively low temperature.

Table 1. Maximum in-line transmittances of $\mathrm{Y}_{2} \mathrm{O}_{3}$ transparent ceramics prepared by SPS sintering at visible light and infrared band. 


\begin{tabular}{|lllllll|}
\hline Samples & $\begin{array}{l}\text { Sintering } \\
\text { temperature }\end{array}$ & Thickness & Sintering aids & Visible & Infrared & Ref \\
\hline Dy: $\mathrm{Y}_{2} \mathrm{O}_{3}$ & $1550^{\circ} \mathrm{C}$ & $1 \mathrm{~mm}$ & $\mathrm{LiF}$ & $75.0 \%$ & $80.1 \%$ & {$[37]$} \\
\hline $\mathrm{Sm}: \mathrm{Y}_{2} \mathrm{O}_{3}$ & $1450^{\circ} \mathrm{C}$ & $1.7 \mathrm{~mm}$ & $\mathrm{LiF}$ & $75.9 \%$ & $80.2 \%$ & {$[36]$} \\
\hline $\mathrm{Y}_{2} \mathrm{O}_{3}$ & $1400^{\circ} \mathrm{C}$ & $1 \mathrm{~mm}$ & No & $47.5 \%$ & $75.2 \%$ & {$[38]$} \\
\hline & $1600^{\circ} \mathrm{C}$ & $1 \mathrm{~mm}$ & No & $63.4 \%$ & $82.2 \%$ & \\
\hline $\mathrm{Y}_{2} \mathrm{O}_{3}$ & $1400^{\circ} \mathrm{C}$ & $2.5 \mathrm{~mm}$ & No & - & $77.9 \%$ & {$[40]$} \\
\hline & $1500^{\circ} \mathrm{C}$ & $2.5 \mathrm{~mm}$ & No & - & $82.0 \%$ & \\
\hline $\mathrm{Y}_{2} \mathrm{O}_{3}$ & $1300^{\circ} \mathrm{C}$ & $3.5 \mathrm{~mm}$ & No & $0 \%$ & $62.1 \%$ & {$[39]$} \\
\hline $\mathrm{Y}_{2} \mathrm{O}_{3}$ & $1300^{\circ} \mathrm{C}$ & $1 \mathrm{~mm}$ & No & $65.9 \%$ & $80.8 \%$ & This work \\
\hline
\end{tabular}

Furthermore, the mechanical properties are very important in mechanical industrial applications of transparent ceramics [41]. According to the Hall-Petch relationship, the strength of the material is inversely proportional to the square root of the average grain size [42]. Therefore, reducing the grain size is of great significance for improving the mechanical properties of transparent ceramics. As the average grain size of $\mathrm{Y}_{2} \mathrm{O}_{3}$ transparent ceramic prepared at the optimal sintering temperature $\left(1300{ }^{\circ} \mathrm{C}\right)$ is only 0.7 $\mu \mathrm{m}$, we have measured the mechanical properties of the sample. The main factors determining the Vickers hardness of $\mathrm{Y}_{2} \mathrm{O}_{3}$ ceramic are grain size and relative density. Moreover, due to the indentation size effect, the greater the load during the test, the lower the hardness [43]. Zhang et al. calculated the theoretical hardness value of $\mathrm{Y}_{2} \mathrm{O}_{3}$ based on the first-principles study to be 7.7GPa [44]. Serivalsatit et al. have prepared $\mathrm{Y}_{2} \mathrm{O}_{3}$ transparent ceramics with a relative density of about $99.8 \%$. Under a loading force of $0.5 \mathrm{~N}$, the hardnesses of the samples with an average grain size of $0.34 \mu \mathrm{m}$ and $328 \mu \mathrm{m}$ were measured to be 7.2 and $9.1 \mathrm{GPa}$, respectively [45]. In the present work, the $\mathrm{Y}_{2} \mathrm{O}_{3}$ transparent ceramic sintered at $1300{ }^{\circ} \mathrm{C}$ shows a Vickers hardness of $9.0 \mathrm{GPa}$, exceeding the theoretical value, which is mainly caused by the strengthening of ultra-fine grains (average grain size: $0.7 \mu \mathrm{m}$ ). The indentation fracture toughness $\left(K_{I C}\right)$ was calculated from the crack length that formed at the corners of the indentations using the method proposed by Marshall et al. [46]. The estimated $K_{/ C}$ is about $1.30 \mathrm{MPa} \cdot \mathrm{m}^{1 / 2}$, which is comparable to that of $\mathrm{Y}_{2} \mathrm{O}_{3}$ transparent ceramic $\left(1.39 \mathrm{MPa} \cdot \mathrm{m}^{1 / 2}\right)$ with ultra-fine grains prepared by Serivalsatit et al.

\section{Conclusions}

In summary, using the lab-made $\mathrm{Y}_{2} \mathrm{O}_{3}$ nanorod powders as the starting materials, ultrafine-grained $\mathrm{Y}_{2} \mathrm{O}_{3}$ transparent ceramic with good optical and mechanical properties was fabricated successfully at a relatively low temperature via SPS. An improving densification mechanism involving particle fracture and rearrangement at $600{ }^{\circ} \mathrm{C} / 50 \mathrm{MPa}$ is revealed. Specifically, the following results were obtained: 
1) Colourless $\mathrm{Y}_{2} \mathrm{O}_{3}$ transparent ceramic with suppressed grain growth (average grain size is about 0.7 $\mu \mathrm{m}$ ) was obtained successfully from nanorod powders, while the corresponding ceramic from nearspherical nanocrystalline powders is not dense enough and opaque.

2) The transparent ceramic shows maximum transmittances of $65.9 \%$ and $80.8 \%$ in visible light and infrared wavelength ranges, respectively. The Vickers hardness and fracture toughness are $9.0 \mathrm{GPa}$ (theoretical hardness value of $\mathrm{Y}_{2} \mathrm{O}_{3}$ is $7.7 \mathrm{GPa}$ ) and $1.30 \mathrm{MPa} \cdot \mathrm{m}^{1 / 2}$, respectively.

3) The high sintering efficiency of nanorod powders originates from its high specific surface area and unique sintering mechanism involving the fracture and rearrangement of nanorods.

4) The unique sintering process can be divided into three stages: (i) In the primary sintering stage, the deviatoric stress accumulates with the increase of sintering pressure. (ii) The nanorod particles can fracture and rearrange when the sintering temperature reaches $600^{\circ} \mathrm{C}$, which associates with the further increase of compact density and decrease of initial particle size. (iii) The grain grows associating with the further densification of compact, in the last sintering stage.

\section{Declarations}

\section{Acknowledgment}

This work is supported by the National Natural Science Foundation of China (No.11802042, No. 51672100), the Key Research and Development Program of Sichuan Provence (2020YFG0192) and International Cooperation Project of Guangdong Province (No.2019A050510049).

\section{References}

1. Wang SF, Zhang J, Luo DW, Gu F, Tang DY, Dong ZL, Tan GEB, Que WX, Zhang TS, Li S, Kong LB. Transparent ceramics: Processing, materials and applications. Prog Solid State Ch 2013, 41: 20-54.

2. Ling J, Zhou Y, Xu W, Lin H, Lu S, Wang B, Wang K. Red-emitting YAG: Ce, Mn transparent ceramics for warm WLEDs application. J Adv Ceram 2020, 9: 45-54.

3. Li X, Snetkov IL, Yakovlev A, Liu Q, Liu X, Liu Z, Chen P, Zhu D, Wu L, Yang Z. Fabrication and performance evaluation of novel transparent ceramics $\mathrm{RE}: \mathrm{Tb}_{3} \mathrm{Ga}_{5} \mathrm{O}_{12}(\mathrm{RE}=\mathrm{Pr}, \mathrm{Tm}$, Dy) toward magnetooptical application. J Adv Ceram 2021, 10: 271-278.

4. Liu Z, Toci G, Pirri A, Patrizi B, Feng Y, Wei J, Wu F, Yang Z, Vannini M, Li J. Fabrication, microstructures, and optical properties of $\mathrm{Yb}$ : $\mathrm{Lu}_{2} \mathrm{O}_{3}$ laser ceramics from co-precipitated nano-powders. $\mathrm{J}$ Adv Ceram 2020, 9: 674-682.

5. Chen Z, Sun X, Shang Y, Xiong K, Xu Z, Guo R, Cai S, Zheng C. Dense ceramics with complex shape fabricated by 3D printing: A review. J Adv Ceram 2021, 10: 195-218. 
6. Permin DA, Boldin MS, Belyaev AV, Balabanov SS, Koshkin VA, Murashov AA, Ladenkov IV, Lantsev EA, Smetanina $\mathrm{KE}$, Khamaletdinova NM. IR-transparent $\mathrm{MgO}-\mathrm{Gd}_{2} \mathrm{O}_{3}$ composite ceramics produced by selfpropagating high-temperature synthesis and spark plasma sintering. J Adv Ceram 2021, 10: 237-246.

7. Kong LB, Huang Y, Que W, Zhang T, Li S, Zhang J, Dong Z, Tang D. Transparent ceramics. Springer: 2015.

8. Xu H, Zou J, Wang W, Wang H, Ji W, Fu Z. Densification mechanism and microstructure characteristics of nano-and micro-crystalline alumina by high-pressure and low temperature sintering. J Eur Ceram Soc 2021, 41: 635-645.

9. Yavetskiy R, Balabanov A, Parkhomenko S, Kryzhanovska O, Doroshenko A, Mateychenko P, Tolmachev A, Li J, Jiang N, Gheorghe L. Effect of starting materials and sintering temperature on microstructure and optical properties of $\mathrm{Y}_{2} \mathrm{O}_{3}: \mathrm{Yb}^{3+} 5$ at\% transparent ceramics. J Adv Ceram 2021, 10: 49-61.

10. Chaim R, Levin M, Shlayer A, Estournes C. Sintering and densification of nanocrystalline ceramic oxide powders: a review. Adv Appl Ceram 2013, 107: 159-169.

11. Lavi R, Jackel S, Tal A, Lebiush E, Tzuk Y, Goldring S. $885 \mathrm{~nm}$ high-power diodes end-pumped Nd: YAG laser. Opt Commum 2001, 195: 427-430.

12. Maglia F, Tredici IG, Anselmi-Tamburini U. Densification and properties of bulk nanocrystalline functional ceramics with grain size below 50nm. J Eur Ceram Soc 2013, 33: 1045-1066.

13. Lupei V, Lupei A, Gheorghe C, Ikesue A. Comparative high-resolution spectroscopy and emission dynamics of Nd-doped GSGG crystals and transparent ceramics. J lumin 2008, 128: 885-887.

14. Lavi R, Jackel S. Thermally boosted pumping of neodymium lasers. Appl Opts 2000, 39: 3093-3098.

15. Lavi R, Jackel S, Tzuk Y, Winik M, Lebiush E, Katz M, Paiss, I. Efficient pumping scheme for neodymium-doped materials by direct excitation of the upper lasing level. Appl Opts 1999, 38: 7382-7385.

16. Huang Z, Deng J, Wang H, Zhang Y, Duan J, Tang Z, Yang Y, He D, Qi J, Lu T. A new method for the preparation of transparent $\mathrm{Y}_{2} \mathrm{O}_{3}$ nanocrystalline ceramic with an average grain size of $20 \mathrm{~nm}$. Scripta Mater 2020, 182: 57-61.

17. Nosrati H, Sarraf-Mamoory R, Emameh RZ, Le DQS, Perez MC, Bünger CE. Low temperature consolidation of hydroxyapatite-reduced graphene oxide nano-structured powders. Mater Adv 2020, 1: 1337-1346.

18. Patterson A. The Scherrer formula for X-ray particle size determination. Phys Rev 1939, 56: 978. 
19. Kumar RS, Priyanka KS, Khanra A, Johnson R. A novel approach of synthesizing nano $\mathrm{Y}_{2} \mathrm{O}_{3}$ powders for the fabrication of submicron IR transparent ceramics. Ceram Int 2021, 47: 16986-16999.

20. Yang H, Li L, Li Y, Shen B, Kang Y, Zhao L, Li J, Dong Y, Li J. Unveiling exceptional sinterability of ultrafine a-Al $\mathrm{O}_{3}$ nanopowders. J Materiomics 2021, 7: 837-844.

21. Huang Z, Qi J, Zhou M, Gong Y, Shi Q, Yang M, Han W, Cao Z, Peng S, Lu T. A facile solvothermal method for high-quality $\mathrm{Gd}_{2} \mathrm{Zr}_{2} \mathrm{O}_{7}$ nanopowder preparation. Ceram Int 2018, 44:1334-1342.

22. Riman RE, Suchanek WL, Byrappa K, Chen C-W, Shuk P, Oakes CS. Solution synthesis of hydroxyapatite designer particulates. Solid State Ionics 2002, 151: 393-402.

23. Huang Z, Deng J, Wang H, Zhang Y, Duan J, Tang Z, Cao Z, Qi J, He D, Lu T. Fast low-temperature densification of translucent bulk nanograin $\mathrm{Gd}_{2} \mathrm{Zr}_{2} \mathrm{O}_{7}$ ceramics with average grain size below $10 \mathrm{~nm}$. J Alloy Compd 2020, 830: 154617-27.

24. Razavi RS, Ahsanzadeh-Vadeqani M, Barekat M, Naderi M, Hashemi SH, Mishra AK. Effect of sintering temperature on microstructural and optical properties of transparent yttria ceramics fabricated by spark plasma sintering. Ceram Int 2016, 42: 7819-7823.

25. Liu K, He D, Wang H, Lu T, Li F, Zhou X. High-pressure sintering mechanism of yttrium aluminum garnet $\left(\mathrm{Y}_{3} \mathrm{Al}_{5} \mathrm{O}_{12}\right)$ transparent nanoceramics. Scripta Mater 2012, 66: 319-322.

26. Wang HM, Jiang JS, Huang ZY, Chen Y, Liu K, Lu ZW, Qi JQ, Li F, He DW, Lu TC, Wang QY. Determination of the compressive yield strength for nano-grained YAG transparent ceramic by XRD analysis. J Alloy Compd 2016, 671: 527-531.

27. Zhang J, Lu T, Chang X, Jiang S, Wei N, Qi J. Yield strength of transparent $\mathrm{MgAl}_{2} \mathrm{O}_{4}$ nano-ceramic at high pressure and temperature. Nanoscale Res Lett 2010, 5: 1329-32.

28. Gerward L, Mo/rup S, Topso/e H. Particle size and strain broadening in energy-dispersive x-ray powder patterns. J Appl Phys 1976, 47: 822-825.

29. Williamson G, Hall W. X-ray line broadening from filed aluminium and wolfram. Acta Metall 1953, 1: 22-31.

30. Zhang L, Yang J, Yu H, Pan W. High performance of La-doped $\mathrm{Y}_{2} \mathrm{O}_{3}$ transparent ceramics. J Adv Ceram 2020, 9: 493-502.

31. Wang Y, Lu T, Gong L, Qi J, Wen J, Yu J, Pan L, Yu Y, Wei N. Light extinction by pores in AlON ceramics: the transmission properties. J Phys D Appl Phys 2010, 43: 275403.

32. Apetz R, Van Bruggen MP. Transparent alumina: a light-scattering model. J Am Ceram Soc 2003, 86 : 480-486. 
33. Jiang $S$, Lu T, Long Y, Chen J. Ab initio many-body study of the electronic and optical properties of $\mathrm{MgAl}_{2} \mathrm{O}_{4}$ spinel. J Appl Phys 2012, 111: 043516-043523.

34. Wang C, Zhao Z. Transparent $\mathrm{MgAl}_{2} \mathrm{O}_{4}$ ceramic produced by spark plasma sintering. Scripta Mater 2009, 61: 193-196.

35. Qi J, Huang Z, Zhou M, Peng S, Ma N, Lu T. Transparent sub-mircon $\mathrm{Gd}_{2} \mathrm{Zr}_{2} \mathrm{O}_{7}$ ceramic prepared by spark plasma sintering using nanocrystalline powders. J Eur Ceram Soc 2018, 38: 2256-2258.

36. Hu Z, Xu X, Wang J, Liu P, Li D, Wang X, An L, Zhang J, Xu J, Tang D. Spark plasma sintering of Sm ${ }^{3+}$ doped $\mathrm{Y}_{2} \mathrm{O}_{3}$ transparent ceramics for visible light lasers. Ceram Int 2017, 43: 12057-12060.

37. Hu Z, Xu X, Wang J, Liu P, Li D, Wang X, Zhang J, Xu J, Tang D. Fabrication and spectral properties of Dy: $\mathrm{Y}_{2} \mathrm{O}_{3}$ transparent ceramics. J Eur Ceram Soc 2018, 38: 1981-1985.

38. Park CW, Lee JH, Kang SH, Park JH, Kim HM, Kang HS, Lee H, Lee JH, Shim KB. Characteristics of $\mathrm{Y}_{2} \mathrm{O}_{3}$ transparent ceramics rapidly processed using spark plasma sintering. J Ceram Process Res 2017, 18: 183-187.

39. Irom E, Zakeri M, Ahangari Hossein Zadeh AS, Safian S, Rahbari A. Low-pressure fabrication of IRtransparent $\mathrm{Y}_{2} \mathrm{O}_{3}$ via spark plasma sintering. Micro Nano Lett 2016, 11: 688-691.

40. Ahmadi B, Reza SR, Ahsanzadeh-Vadeqani M, Barekat M. Mechanical and optical properties of spark plasma sintered transparent $\mathrm{Y}_{2} \mathrm{O}_{3}$ ceramics. Ceram Int 2016, 42: 17081-17088.

41. Manning W, Hunter J, Powell B. Elastic properties of polycrystalline yttrium oxide, dysprosium oxide, holmium oxide, and erbium oxide: room temperature measurements. J Am Ceram Soc 1969, 52: 436-442.

42. Naik SN, Walley SM. The Hall-Petch and inverse Hall-Petch relations and the hardness of nanocrystalline metals. J Mater Sci 2020, 55: 2661-2681.

43. Nix WD, Gao H. Indentation size effects in crystalline materials: a law for strain gradient plasticity. J Mech Phys Solids 1998, 46: 411-425.

44. Zhang X, Gui W, Zeng Q. First-principles study of structural, mechanical, and thermodynamic properties of cubic $\mathrm{Y}_{2} \mathrm{O}_{3}$ under high pressure. Ceram Int 2017, 43: 3346-3355.

45. Serivalsatit K, Kokuoz B, Yazgan-Kokuoz B, Kennedy M, Ballato J. Synthesis, Processing, and properties of submicrometer-grained highly transparent yttria ceramics. J Am Ceram Soc 2010, 5: 13201325.

46. Anstis G, Chantikul P, Lawn BR, Marshall D. A critical evaluation of indentation techniques for measuring fracture toughness: I, direct crack measurements. J Am Ceram Soc 1981, 64:533-538. 


\section{Figures}
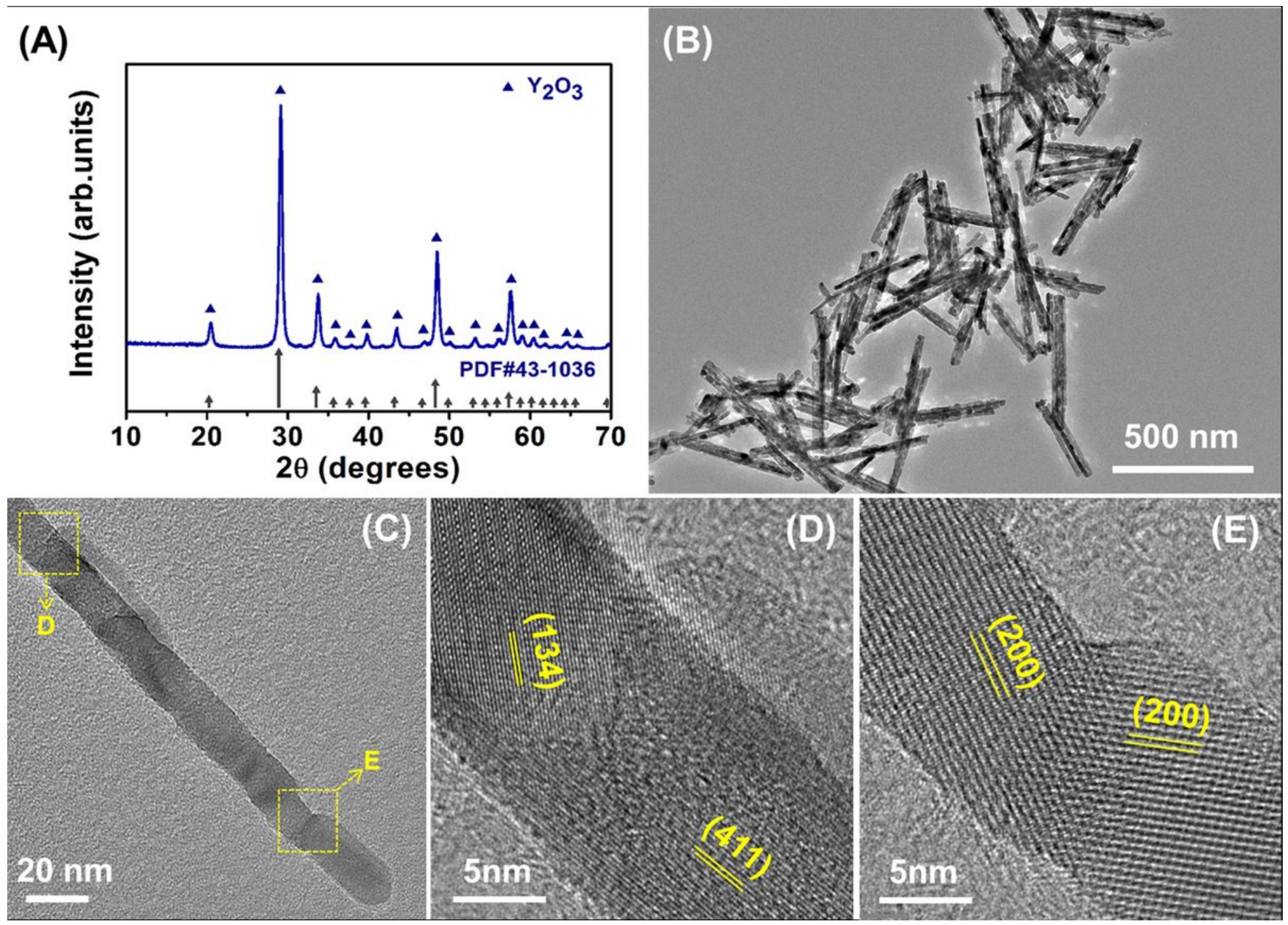

Figure 1

XRD pattern of the $\mathrm{Y} 203$ powders calcined at $800^{\circ} \mathrm{C}(\mathrm{A})$. TEM images of the $\mathrm{Y} 2 \mathrm{O} 3$ nanocrystalline powders prepared by the solvothermal method $(B, C)$. High-resolution TEM (HRTEM) images taken from different areas in Fig. 1C (D, E). 


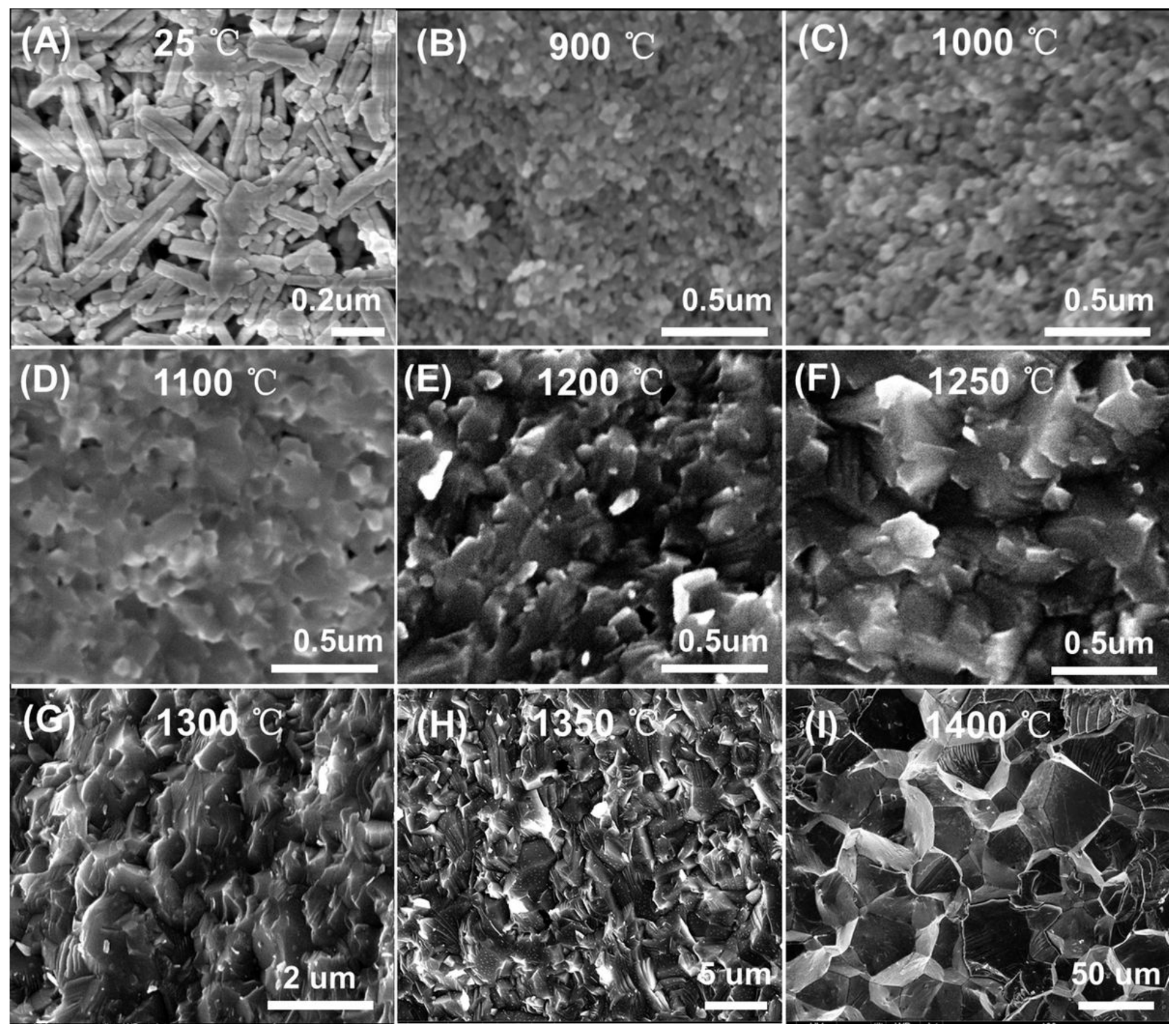

Figure 2

Fracture surface images of the green body from nanorod powders compacted at $50 \mathrm{MPa}$ and ceramics sintered at $900-1400^{\circ} \mathrm{C}$. 
(A)
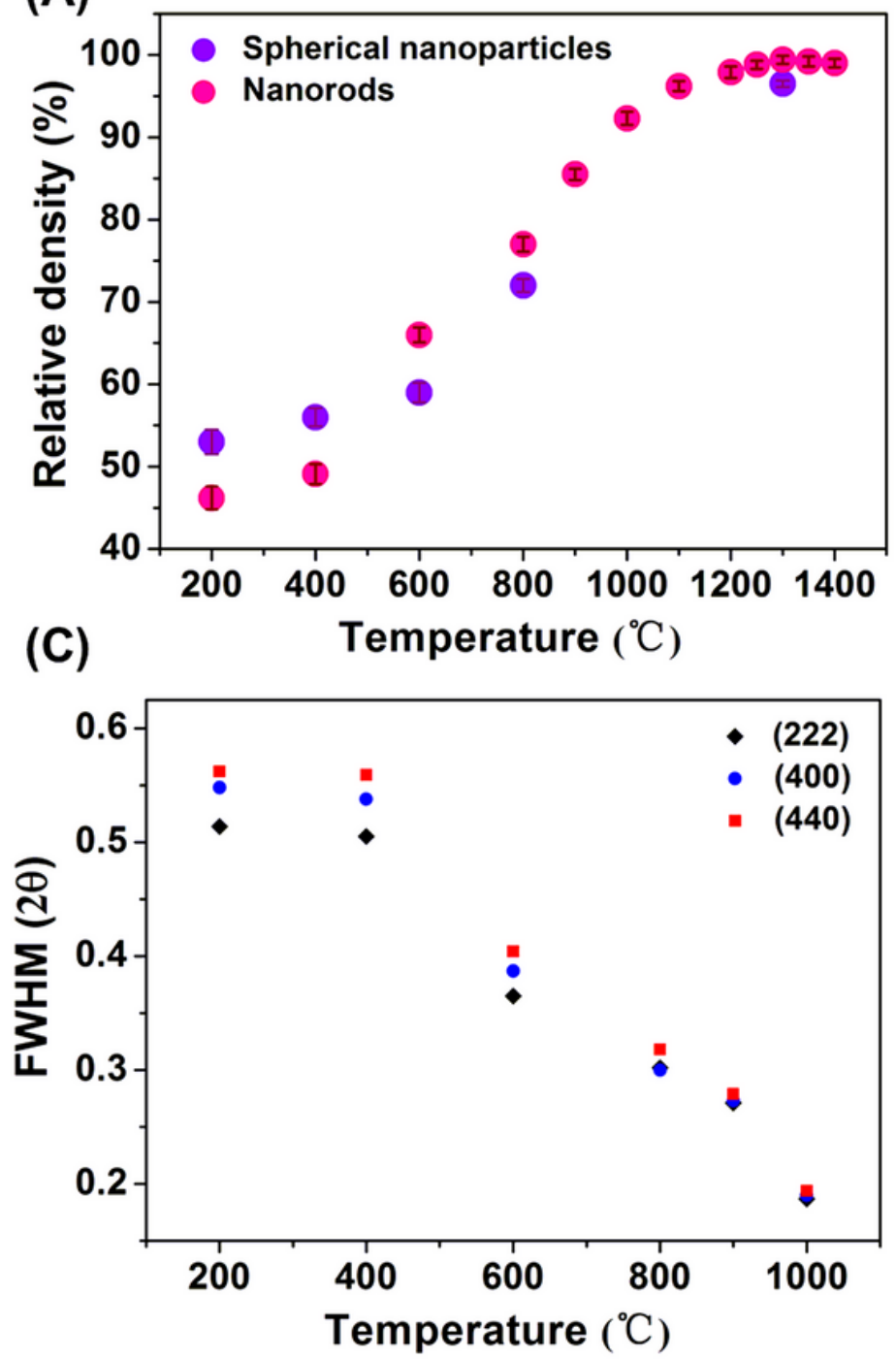

(B)
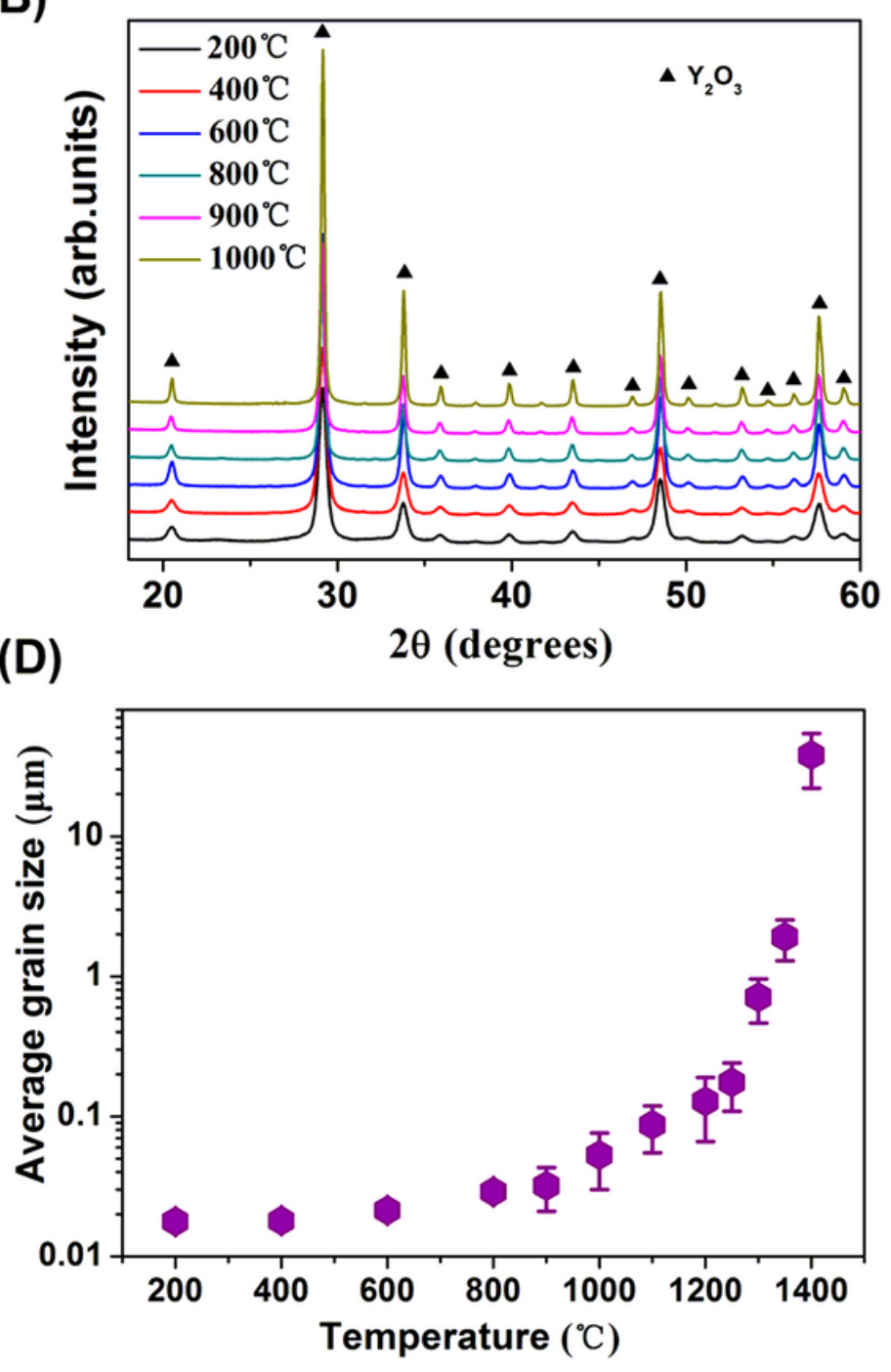

Figure 3

Relative densities of as-sintered ceramics from nanorods and near-spherical nanocrystalline powders (A). XRD patterns of ceramics from nanorods sintered at the temperature range of $200-1000{ }^{\circ} \mathrm{C}(\mathrm{B})$. FWHMs of diffraction peaks of (222), (400), and (440) as a function of sintering temperature (C). Average grain size of ceramic from nanorods as a function of sintering temperature (D). 

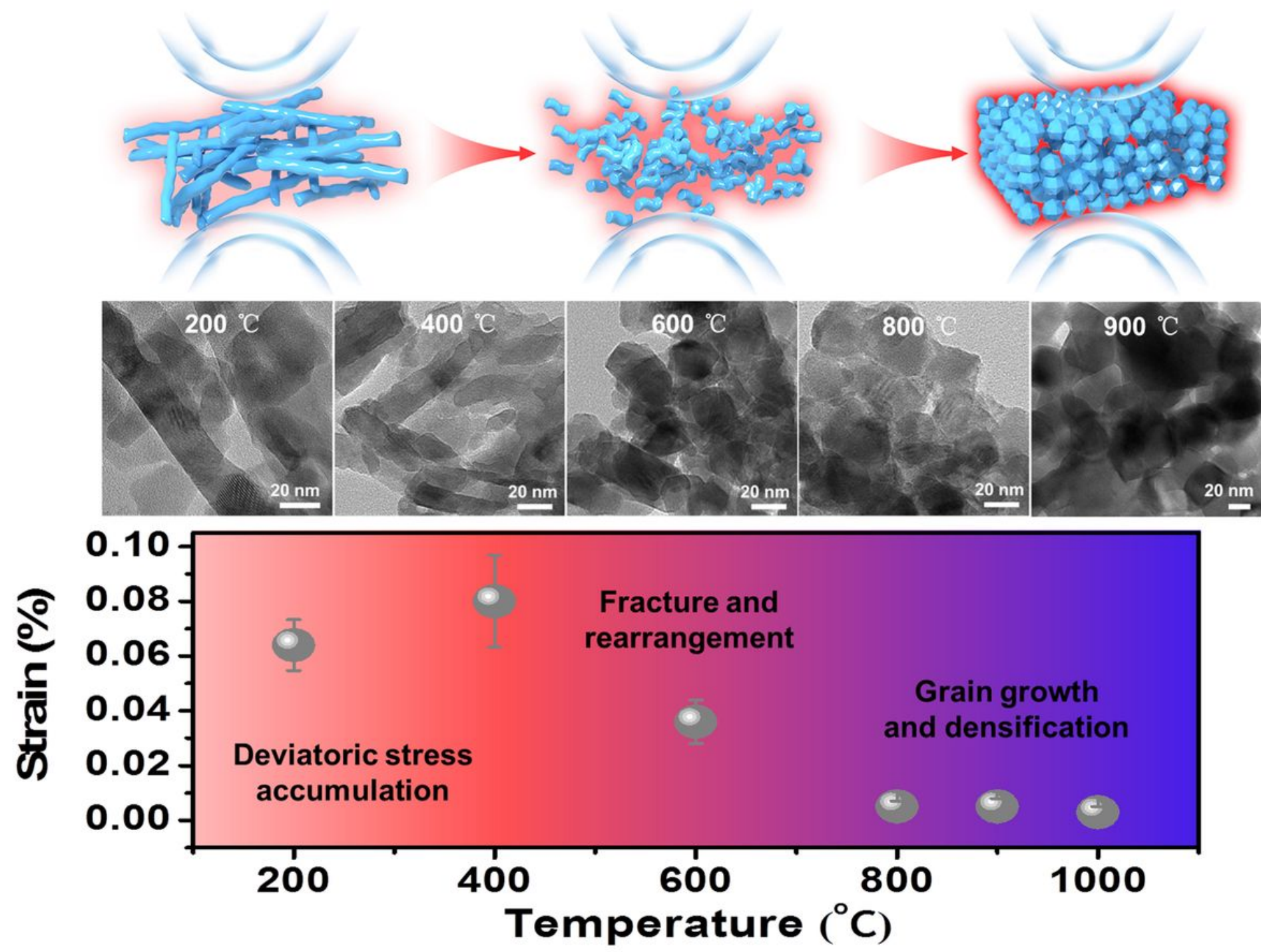

Figure 4

TEM images of the ceramics from nanorod powders sintered at $200-900{ }^{\circ} \mathrm{C}$. Residual strain calculated from whole-pattern-fitted XRD data as a function of sintering temperature was also presente. Combining microstructure evolution and residual stress analysis, a schematic is plotted to show the specific densification mechanism of nanorod powders by fracture/rearrangement. 


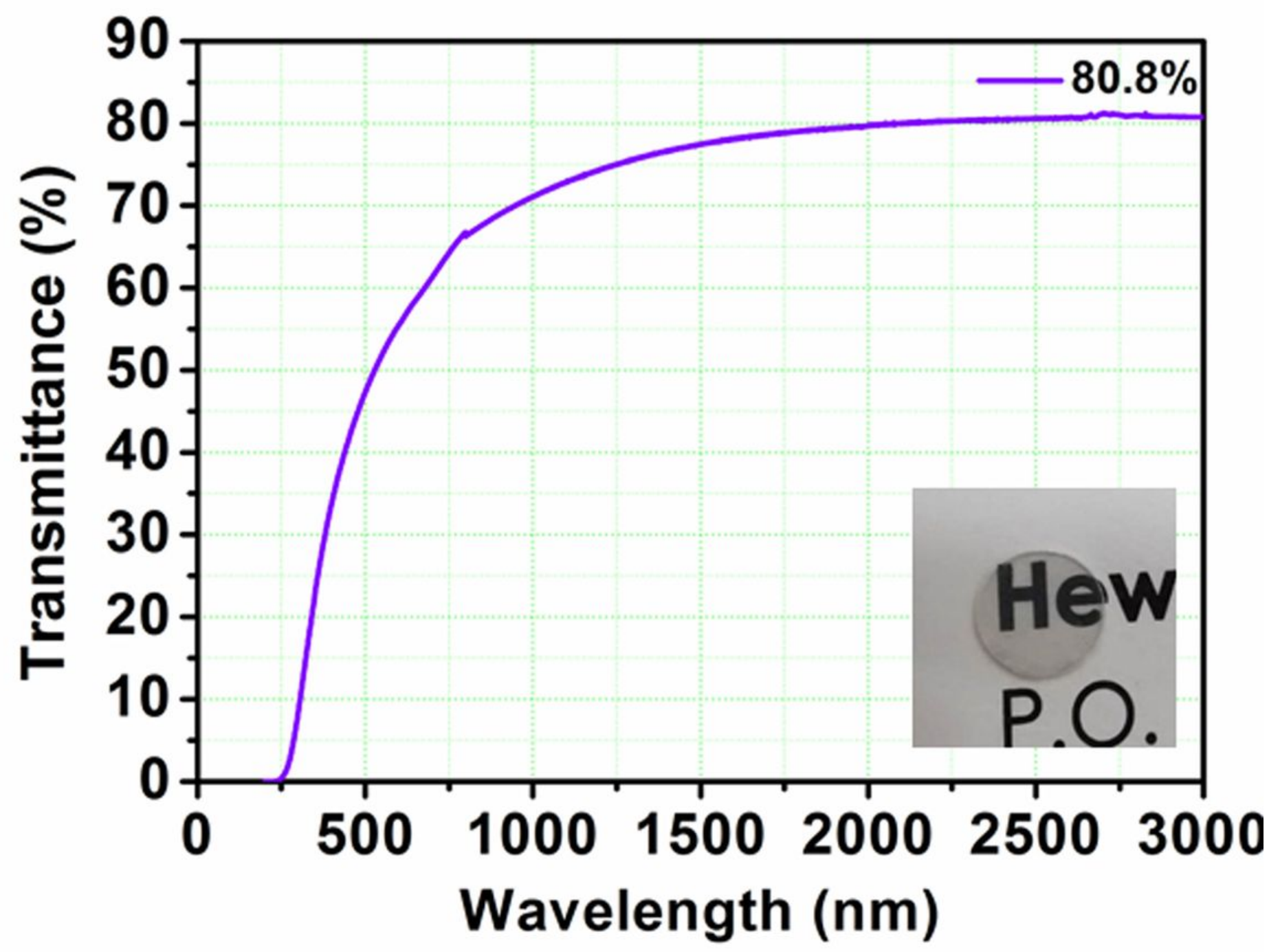

Figure 5

In-line transmittance profile of the $\mathrm{Y} 203$ transparent ceramic sintered at $1300^{\circ} \mathrm{C}$ and annealed at 1000 ${ }^{\circ} \mathrm{C}$. The inset is the optical image of the corresponding ceramic.

\section{Supplementary Files}

This is a list of supplementary files associated with this preprint. Click to download.

- Supplementary.docx 